\title{
Cuerpos, educaciones y géneros. Reflexiones sobre la anestesia y lo sensible en pedagogía
}

Bodies, educations and genders. Reflections on anesthesia and sensory pedagogy

\author{
Jordi Planella Ribera \\ e-mail: jplanella@uoc.edu \\ Universitat Oberta de Catalunya. España
}

Joxé Jiménez Jiménez

e-mail: joxe.jimenez@ehu.eus Euskal Herriko Unibertsitatea. España

\author{
Lucero Alexandra Ruiz Ortega \\ e-mail: lucero.ruiz@udea.edu.co \\ Universidad de Antioquia. Colombia
}

\section{Resumen}

Este trabajo tiene por objetivo presentar una reflexión sobre diferentes cuestiones relacionadas con las formas de transmitir saberes vinculados con el cuerpo, la educación y la perspectiva de género en el contexto universitario, y de forma concreta en la formación de profesionales de la educación. Este trabajo se inscribe en un programa de investigación más amplio que lleva por título «Pedagogías Sensibles» y que lo autores venimos desarrollando en los últimos años. Lo que presentamos es una revisión de algunas de las ideas elaboradas y sistematizadas a lo largo de dicho programa de investigación y que tienen como principal objetivo clarificar las formas como se transmiten, en la universidad, las cuestiones vinculadas al cuerpo, al género y a la educación. Para ello nos hemos servido de diferentes autores que son relevantes por su producción y por determinadas formas de pensar y entender este tipo de educación. Con dichos autores se ha establecido un diálogo, y de forma especial con Valeria Flores, Gloria Anzaldúa y Gayatri Ch. Spivak.

Palabras clave: pedagogía sensible; género; cuerpo; queer; postcolonialidad

\section{Abstract}

This work aims to present a reflection on different issues related to the ways of transmitting knowledge related to the body, education and gender perspective in the university context, and specifically in the training of education professionals. This work is part of a broader research program that is entitled "Sensitive Pedagogies" and that authors have been developing in recent years. What we present is a review of some of the ideas elaborated and systematized throughout this research program and whose main objective is to clarify the ways in which, in the university, the issues related to the body, gender and education are transmitted. For this we have used different authors who are relevant for their production and for certain ways of thinking and understanding this type of education. With these authors a dialogue has been established, and in a special way with Valeria Flores, Gloria Anzaldúa and Gayatri Ch. Spivak.

Keywords: sensitive pedagogy; gender; body; queer, postcoloniality

Recibido / Received: 15-01-2019

Aceptado / Accepted: 20-03-2019

Publicación en línea / Published online: 01-07-2019

Cómo referenciar este artículo / How to reference this article:

Planella Ribera, J., Jiménez Jiménez, J., \& Ruiz Ortega, L. A. (2019). Cuerpos, educaciones y géneros. Reflexiones sobre la anestesia y lo sensible en pedagogía. Tendencias Pedagógicas, 34, pp. 5-15. doi: 10.15366/tp2019.34.002 


\section{Apertura}

En un breve ensayo editado en forma de opúsculo bajo el título Afectos, pedagogias, infancias y heterenormatividad. Reflexiones sobre el daño, Val Flores (2016), escritora, maestra y activista queer se hace un serie de preguntas que nos sirven para arrancar nuestro trabajo:

«¿Como docentes nos podemos imaginar promoviendo imágenes de cariño entre varones, nenas con pelo corto, chicos con vestidos o paseando bebés, niñas que quieran desarrollar la musculatura, de familias con madres lesbianas, gays o trans, por ejemplo? ¿Podemos imaginar una sala de maestrxs donde las conversaciones sobre sexualidad gay o de identidad trans no sean motivo de burla permanente o donde la sexualidad lesbiana no sea un acallamiento indisoluble?» (p. 8).

Nos parece más que evidente que en educación se trata de pensar y buscar determinadas preguntas que orienten el trabajo cotidiano, pero también el trabajo de investigación sobre las formas de transmitir. A la estela de lo dicho, el objetivo principal de este trabajo es compartir los resultados de diferentes experiencias de transmisión e investigación de saberes vinculados al campo de la educación, que podemos calificar como «pedagogías sensibles» o «pedagogías corporales» y que, desde nuestro punto de vista, se convierten en fundamentales en la formación de profesionales del campo de la educación. Este tipo de pedagogías, podemos entenderlas también como pedagogías autónomas, como pedagogías que no se encuentran sometidas a determinadas posiciones hegemónicas en las que se determina y norma lo que se puede y lo que no se puede hacer. Tal vez se traten de pedagogías que puedan ser entendidas como «segundas pieles», como aquellas que presentan o representan otras formas de pensar, entender y ejercer la educación. Las segundas pieles tienen esa capacidad de cubrir y descubrir, de transformar, de performar determinadas realidades, y eso es relevante en un ejercicio que se propone ofrecer otras miradas a la educación simbólica del cuerpo y a las posibilidades de ejercer y vivir la diferencia corporal (en especial de género) como algo que no debe «esconderse» bajo el manto de la normalidad (y de la normalización pedagógica). La educación simbólica del cuerpo (Leib) se contrapone a la educación física de los cuerpos (Körper). En esta última se privilegia una determinada visión y concepción de los cuerpos basada en las perspectivas anatómicas y fisiológicas del mismo. La dimensión simbólica posibilita una hermenéutica corporal desde la palabra encarnada y a partir de la comunicación del ser humano en todas sus dimensiones (corpografía).

Figura 1.

Corpografias

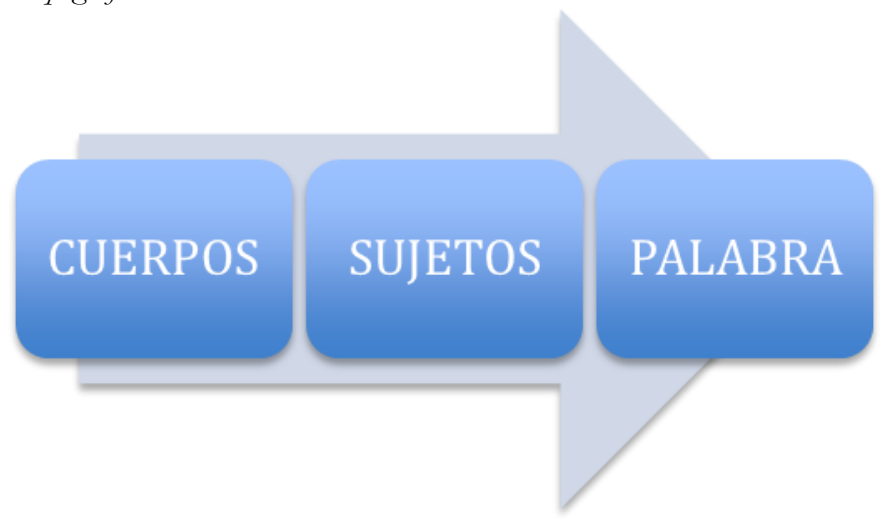

Fuente: Elaboración propia

Las cuestiones que aquí aparecen se enfocan desde la «experiencia» y tienen que ver con lo que nos propone Foucault (2013):

«no hay libro que no haya escrito sin, al menos en parte, una experiencia directa, personal. $\mathrm{He}$ tenido una relación personal, compleja, con la locura y con la Institución psiquiátrica. He tenido con la enfermedad y con la muerte también una cierta relación. He escrito sobre el Nacimiento de la clínica y la introducción de la muerte en el saber médico en un momento en que esas cosas tenían cierta importancia para mí» (p.187). 
Uno de los elementos clave de este tipo de pedagogía es la no separación entre lo que estudian los autores que están vinculados a ella y sus propias vidas. Y en ello insiste Vicenzo Costa (2018) al decir que «conviene, en suma, iluminar la radicación de la experiencia educativa en la vida, a fin de entenderla como interna a su movimiento» (p. 30). Conectar la educación con la vida, un reto relevante y nada fácil para poder pensar las formas de transmisión contemporáneas. Se trata de una pedagogía encarnada, de algo en lo que insiste claramente Tolokonnikova (2018) cuando dice que: «Creo en la unión de la teoría y la práctica, de las palabras y los hechos. Procuro no hablar demasiado sin hacer algo a medias (...) En el principio existía la palabra, pero como todos sabemos, los hechos le siguieron de cerca» (p. 20). Nos parece que este hecho, la conexión entre el cuerpo y la palabra, entre lo dicho y lo vivido, se convierte en algo realmente central. De ello se desprende la idea de la corpografía, de una vida vivida desde y con el cuerpo y en parte de pensarnos como seres bioculturales o encarnados. Podemos entender que «la encarnación consiste en el hecho de tener carne, más aún, de ser carne. Los seres encarnados no son, pues, cuerpos inertes que no siente ni experimentan nada, sin conciencia ni de ellos mismos ni de las cosas» (Henry, 2018, p. 9).

\section{Método}

Metodológicamente hablando, hemos organizado el trabajo a partir de tres grandes cuestiones: a) preguntas de investigación y objetivos, b) fuentes de información y corpus textual, y finalmente c) el análisis de los resultados. Ello permite disponer de una perspectiva más amplia porqué implica partir de las preguntas que guiarán el proceso de investigación, el manejo de los distintos corpus textuales como fuentes de información y el posterior ejercicio hermenéutico que desvela sus claves.

La investigación que presentamos ha tenido por objetivo situar y analizar las pedagogías que se relacionan con el cuerpo y el género en tres categorías generales. En este sentido, algunas de la preguntas que han guiado la investigación han sido: ¿puede la pedagogía transmitir en la formación de profesionales de la educación cuestiones vinculadas a lo sensible? ¿Lo sensible tiene sentido en un contexto donde priman los aprendizajes y las transmisiones operadas desde lo intelectual? ¿Quién decide lo que es y lo que no es transmisible en relación a los cuerpos y los géneros? ¿Existen patrones hetero-normativos que imperan en los modelos pedagógicos?

Hemos contado con diferentes fuentes documentales para el desarrollo del trabajo. Se han manejado libros (monografías como fuentes primarias y secundarias) y narrativas de los estudiantes que han participado en alguno de los seminarios de docencia e investigación (en forma de narrativas orales, textos o cartas, o audiovisuales). Para ello partimos de lo que nos proponen Giribuela y Nieto (2010): «las palabras dichas por otros, tanto aquellas que provienen de testimonios como los procedentes de la lecturas del profesional, suelen tener un espacio significativo en los informes sociales» (p. 89). Para esta investigación nos parecía relevante la inclusión de otras voces, voces de estudiantes y de otros investigadores, testimonios de las pedagogías sensibles que hemos estudiado. La cuestión de las narrativas de los propios sujetos se convirtió en algo fundamental, y de ello nos interesaba especialmente «cómo cuentan sus historias los individuos -lo que enfatizan, lo que omiten, su postura como protagonistas o víctimas, la relación que la historia establece entre el que cuenta y el auditoriotodo ello moldea lo que un individuo puede afirmar sobre su propia vida» (Rosenwald y Ochberg, 1993, p. 2). Dichas fuentes han sido analizadas desde la perspectiva del análisis textual. Las informaciones se han recogido a partir de un proceso de investigación-acción en distintos espacios formativos, en especial seminarios. En palabras de Finkel (2008) «el seminario abierto permite que los estudiantes aprendan mediante la indagación mutua. Su forma de proceder, en un sentido amplio, es científica. Su esencia es formular hipótesis y contrastarlas con la experiencia» (p. 86), o tal y como propone Certeau (2006) «un Seminario es un laboratorio común que permite a cada uno de los participantes articular sus prácticas y sus propios conocimientos» (p. 43). Es a través de dicha forma de dar la palabra que se pudieron recoger textos, cartas o palabras que eran en realidad, formas de construcción de distintos saberes sobre la educación.

El análisis de las informaciones se ha desarrollado desde la hermenéutica y situándolo en el campo disciplinar de la Filosofía de la Educación. A partir del marco teórico en el que hemos ubicado la investigación (hermenéutica educativa y narrativa pedagógica) hemos llevado a cabo el análisis de las informaciones recogidas y segmentadas. El trabajo aquí presentado y el análisis de las informaciones se ha desarrollado desde la hermenéutica, situándose en el campo disciplinar de la Filosofía de la Educación. El análisis se ha realizado a partir de tres grandes categorías: 1) Pedagogía Sensible, 2) 
Transdiversidad y 3) Postcolonialidad. Se ha trabajado con unidades de relato que designaban episodios sociales y teorías concretas de las experiencias formativas que han tenido lugar en las asignaturas, seminarios o jornadas. Para la concreción de las categorías se ha tenido en cuenta lo que Duverger (1996) propone: «El trabajo material de examen del texto es casi secundario respecto de la formulación de las categorías que le sirven de marco» (p. 181). Las tres categorías han servido para desarrollar un sistema de interrelación teórica de las formas pedagógicas estudiadas que nos han permitido una comprensión global de los textos y de las diferentes experiencias, y se ha hecho desde la lógica del preguntarse por los problemas que nos rodean y algunas de las respuestas posibles que operan en los procesos transformativos de la realidad.

\section{Acto I: formar desde lo sensible}

La investigación que presentamos permite analizar un tema amplio y en realidad poco definido pero que preocupa e interesa cada vez más a una parte nada despreciable de profesionales vinculados, de una u otra forma, al campo de la educación. La idea de formar desde la perspectiva de lo sensible se alinea con lo que propone Flores (2016): «este trabajo pretende incitar a (re)pensar la infancia en nuestras prácticas pedagógicas como instancia de sexualización, su articulación con los modos heterosexualizados del saber escolar, y cómo los afectos participan de esta pedagogía heteronormativa» (p. 3). Resituar lo normativo en relación a las transmisiones sobre género se convierte en algo fundamental para poder abrir la mirada, para poder acoger el desarrollo de vivencias no atravesadas por cierta hegemonía de las masculinidades en las aulas. Ello implica, necesariamente, pensar y repensar como se forman a los profesionales de la educación: «la acción educativa está condicionada también por el modo en que se educan los educadores» (Brezinka, 2002, p. 18).

La pedagogía sensible puede ser entendida como una forma genérica que incorpora diferentes miradas y perspectivas sobre la educación, y estas perspectivas o dimensiones pueden tener diferentes lecturas y enfoques epistemológicos. Una de las lecturas posibles tiene que ver con el hecho de tener o mantener los sentidos despiertos, no anestesiados. Los sentidos nos relacionan directamente con el cuerpo humano y con determinadas formas de concebirlo y de ejercer, a través de él, la condición de sujeto encarnado. A menudo la pedagogía busca adormir (anestesiar) más que despertar a los sujetos de la educación. En pleno Seminario sobre Corpografiasy Pedagogias Sensibles en la UFGRS (Porto Alegre, Brasil) el año 2015 y visitando el Museo da Historia de la Medicina de Rio Grande do Sul (MUHM), vimos de forma muy clara esta metáfora cuando nos encontrábamos, justamente, en la sala dedicada a la anestesia. Más tarde, al compartirla con los estudiantes, aparecieron muchas sensaciones y experiencias vividas a lo largo de su formación que se podían vincular directamente con aquella imagen de un paciente tumbado en el quirófano, quieto y del que se espera un alto nivel de pasividad. El texto de presentación de dicho seminario anunciaba:

«El seminario Corpografias y Pedagogías Sensibles busca mostrar a los estudiantes el estado de las investigaciones que vinculan las cuestiones corporales (Body Studies) con las praxis pedagógicas. La pedagogía sensible, la educación de la corporeidad u otras formas de denominar algo que más allá de la tecnificación, de la mecanización y la excesiva didactización, busca ofrecer otras miradas a la educación. Dichas miradas intentan ir más allá de lo puramente mecánico, anatómico, físico para atreverse a ofrecer algo del orden pedagógico que no produzca situaciones de anestesia en los dispositivos pedagógicos» Universidade Federal de Rio Grande do Sul» (Porto Alegre, Brasil), [08/2015]

Algunas pedagogías (y sus representantes) siguen soñando con este estado de anestesia de sus estudiantes; una anestesia, que a menudo se traduce en el deseo de hacer desaparecer el cuerpo y todo lo que la condición corporal recuerda a nuestra dimensión de «sujetos de carne». Henry (2018) insistirá en la idea que «los seres encarnados son seres sufrientes, atravesados por el deseo y el temor, que sienten toda una serie de impresiones vinculadas a la carne por cuanto que, constituidas de su sustancia -una sustancia impresional por tanto- comienzan y acaban con lo que ella experimenta» (p. 9). Vinculado a la idea de la anestesia, pero en sus antípodas, encontramos la idea fundamental de resistencia y de otras pedagogías que apuestan por las dimensiones sensibles y corporales de los sujetos. Esta carta de una de las participantes en el citado Seminario es un claro ejemplo de lo que acabamos de plantear: 


\begin{abstract}
«Recuerdo también que una vez llegaron unas muchachas de Saba a darnos una "charla"; ese día nos separaron a las niñas de los niños. No sé qué habrá pasado con ellos, pero a nosotras nos hablaron de la regla y de las toallas que podíamos usar nosotras que éramos más pequeñas. Por la edad que teníamos, se acercaba ese momento de que nos "viniera" y teníamos que estar preparadas para no mancharnos. La idea que me quedaba (o más bien, que nos queda a varias) fue que la regla tenía que esconderse, que era algo sucio y que nos cuidáramos mucho para que nunca apareciera ninguna mancha roja. Esa fue (o es) una de las grandes lecciones que nos dan a las mujeres y que tanto daño nos hacen» (Carta 1).
\end{abstract}

En una dirección parecida, Corazza (2002) nos habla de la «pedagogía del infierno» al hacer referencia a la educación anunciada por Nietzsche, Deleuze y otros malditos afines y apuesta por una pedagogía que se proponga: «la tarea específica de ser capaz de pensar lo impensable, lo intratable, lo imposible, lo no pensado del pensamiento educativo (...) Pensamiento que iguala las verdades recibidas, metamorfosea el valor de las opiniones establecidas, y busca suspender y transvalorar el valor de todos los valores y las verdades» (p. 31).

Retomando la idea de Anestesia (’’ $v \alpha 1 \sigma \theta \eta \sigma i ́ \alpha)$, este es un término de origen griego que significa, justamente, ausencia de sensaciones, ausencia de sensibilidad. El estado de ausencia de dolor, por ejemplo, para las intervenciones quirúrgicas, contrasta con su sentido en el campo educativo. Es así que podríamos hablar de pedagogía anestesiada y de pedagogía anestesiante. La pedagogía anestesiada toma la forma verbal del pasado y se presenta como algo ya concluido; la pedagogía anestesiante toma la forma verbal -activa- de la potencia, del potencial futuro de negación de determinadas subjetividades. Ya sabemos que el fin de la anestesia es la paralización de determinadas partes del cuerpo humano para realizar algún tipo de operación en ellas o con ellas. No despertar al sujeto (con sus pasiones, saberes, placeres, encarnaciones) sino mantenerlo dormido y pasivo -corporalmente hablando-. He aquí el fin de determinadas pedagogías. Se trataría, en definitiva, de no aceptar que el cuerpo podría estar controlado en todos sus aspectos, reemplazado, y haber así alimentado, a menudo sin darse cuenta, el fantasma de un dominio absoluto sobre él. Pasa a ser, entonces, un cuerpo tendido en una camilla, en un quirófano, o bien sentado pasivamente en un pupitre de nuestras universidades, sin moverse, atento y sin molestar. Otra de las cartas de participantes en el Seminario plantea lo siguiente:

\begin{abstract}
«Decido huir de las técnicas del cuerpo. Estudio $5^{\circ}$ curso de primaria en una escuela donde el cuerpo debe, tiene, necesita disciplinarse. Tengo 10 años y hemos dejado atrás la dictadura franquista ¿Por qué seguir con esa parafernalia? ¿Cuándo nos hablarán del cuerpo? ¿Deberemos correr eternamente dando vueltas al patio? Noto que se me desgarra el alma: sé que no quiero ser un número, una pequeña pieza de un gran engranaje, carne destinada a obedecer, a deformarse, a normalizarse. Resistir, resistir frente a muchas cosas, pero sobre todo a la producción uniforme de cuerpos masculinos (las niñas llegaran el siguiente año pero a un curso inferior), blancos, burgueses, limpios, cristianos y no sé cuántas cosas más» (Carta 2).
\end{abstract}

De este sujeto de la educación se espera -sobre todo- que no se mueva, que esté pasivo (¿no es este el significado de paciente en el campo médico?. Un significado que se postula contrario a lo que nos decía Jacques Lacan (1981): «yo hablo con mi cuerpo, y ello sin saberlo. Digo pues siempre más de lo que sé» (p. 101). Y esto, a la pedagogía, le sigue costando de entender y aceptar. Para muchas pedagogías los cuerpos no hablan, son silencio y no gritos desgarrados, y cuando deciden tomar la palabra (prestada, robada o arranque de las manos del poder) la pedagogía se teme lo peor e imagina mundos subversivos, incontrolados y peligrosos.

\title{
4. Acto II: transgredir la norma. ¿Qué pasa con la pedagogía transdiversa?
}

¿Puede, la pedagogía, transgredir algo del orden social, político y educativo ya establecido? A partir de esta pregunta intentaremos organizar diferentes reflexiones en torno a la idea de transdiversidad. Para ello me serviré de experiencias, de discursos encarnados, que van desde Gloria Anzaldúa hasta la vivencia de la transgresión por parte del activista y cantante punk Urko Gato. Dice Ricardo Llamas (1998) que pensar el mundo desde la Teoría Queer es situarse en lo que podemos denominar como «teoría torcida», en una teoría que -ubicada en la línea de fuga de autores como Fernand Deligny, Michel Foucault, Hans G. Gadamer y otros- busca la frontera como espacio de ruptura, como franja 
donde es posible pensar de otro modo (p. 87). Con el paso de los años se ha hecho evidente que hay claras conexiones entre lo que se ha venido denominando teoría queer y la pedagogía (podemos denominar a esta unidad terminológica como Pedagoqueer y de hecho esta denominación ha empezado a cuajar ya en diferentes discursos y publicaciones). Este hecho no siempre ha sido evidente, e incluso ha sido visto y temido como algo extraño (y porque no decirlo: peligroso). Mezclar diversidades sexuales con la educación podía tener un cierto sabor a «peligro», podía hacer pensar a alguien (especialmente con mentalidades cerradas y ultra conservadoras) que poner en manos de educadores la palabra queer era pervertir, era torcer la educación misma, e incluso ha sido visto y temido como algo extraño. Así lo narra Pepe Miralles (2014):

\begin{abstract}
«Realmente me ha importado poco saber las preferencias sexuales del alumnado que he conocido. Contrariamente, en ocasiones sí que he notado que a ciertos estudiantes les ha importado mi preferencia sexual, que manifiesto con naturalidad desde el primer día de clase (...) En las clases que imparto, mis comentarios, aportaciones y análisis están contaminados por mi preferencia sexual. Ser marica es una forma concreta de afrontar la existencia, consecuencia de años de experiencias generalmente relacionadas con la injuria. Ser marica conlleva formas de relacionarse distintas, de percibir y ser percibido. Pero también implica, al menos en mi caso, habitar un espacio fuera de la norma» (p. 155).
\end{abstract}

Pero también es cierto que lo que sucede en los contextos o dispositivos educativos es un claro reflejo de lo que trasluce en la sociedad: estigmatizar, señalar, condenar determinados usos del cuerpo, de los cuerpos que deciden «habitar un espacio fuera de la norma». Lo dice Trujillo (2013): «Ser queer es ser raro, diferente, escaparse de la heteronormatividad, del binarismo de género y sexual. Un chico "afeminado" al que le gusta jugar con sus amigas a la goma en el patio de la escuela o una chica "marimacho" que se pasa las horas dándole patadas a la pelota no encajan en lo que se espera de sus comportamientos como chico y chica respectivamente» (p. 8). La manipulación de los cuerpos (y lo que representan o pueden representar) toma una fuerza especial en las praxis pedagógicas. El cenit (o la imaginación de otras fronteras) de las políticas educativas vinculadas a la diferencia es de una limitación increíble. ¿Qué entienden estas políticas por diferencia? ¿Quién dictamina, otorga, clasifica, distribuye o redistribuye a los sujetos de la educación considerados diferentes? El normalismo (una práctica demasiado extendida en la sociedad contemporánea) ha provocado daños irreparables, ya que ha sido el patrón que ha regido y marcado lo que está permitido y lo que no, el patrón que ha marcado el ritmo de clasificación y ordenación de grupos y sociedades. Para Flores (2016) «Este régimen de conocimiento y desconocimiento que establece la heteronormatividad, construye una distinción radical entre lo que se considera público, admisible en el campo de lo dicho, la autorizado socialmente acerca de cómo deben ser leídos o comprendidos los cuerpos, y lo infame, lo indecible, aquello cuyo sólo nombre produce espanto, indignación, escándalo o corrupción de la estructura social y de las buenas costumbres» (p. 7).

Podemos afirmar con rotundidad que el normalismo está instaurado de forma estable en nuestras sociedades; de hecho es quien las ordena, quien las guía, quien administra su ritmo. En una carta de otro de los participantes en el Seminario de Porto Alegre (2015) se plantea que:

\begin{abstract}
«Mi experiencia en la escuela estuvo marcada por el hecho de tener los pies hacia adentro. Para mis colegas yo era el pie torcido, pie de hoz, entre otros juegos de palabras. El hecho de caminar de forma diferente despertó gracia en los otros niños, y un sentido de corrección en mí. Las discusiones que tuvimos en el seminario posibilitaron trascender el concepto de cuerpo para explorar la dimensión simbólica y convivir con colegas que experimentan de maneras diversas lo cotidiano. Para mí, revisar este hecho a través de los desafíos propuestos en clase, despertó necesidad de repensar las formas como reaccionaba por el hecho de tener pies torcidos en la escuela» (Carta 3).
\end{abstract}

Lo transdiverso se sitúa, no únicamente en el plano de lo sexual (diversidad sexual en un sentido amplio) sino en la idea misma de diversidad corporal, de cuerpos no normativos en todas sus perspectivas. Desde esta posición nos parece interesante poder poner de lado la cuestión de los cuerpos «torcidos» (ejemplo de la Carta 3) con los cuerpos con orientaciones sexuales diversas por la retroalimentación que pueden hacerse estas temáticas al pensar en el sentido profundo de lo trans. Así lo plantea Missé (2018): «Me refiere a que nos han robado la posibilidad de vivir el cuerpo de otra forma, 
que se nos ha impuesto una interpretación única sobre algunas de sus partes. Me refiero a que para explicar nuestro malestar se nos ha dicho que no deberíamos haber nacido en este cuerpo pero que podemos lograr el adecuado con tratamientos hormonales y algunas intervenciones quirúrgicas» (p. 11). Tratar el cuerpo por tener un a «deformidad» física o de otro tipo, conlleva, muchas veces, la praxis violenta del normalismo, de la sujeción (física o moral) a esa norma que regula las formas de ser y estar en sociedad.

Si compartimos la idea de que la orientación sexual y la identidad de género integran aspectos esenciales de la vida de la persona, entenderemos la relevancia de introducir esta perspectiva en las políticas y praxis educativas (Clade, 2014). Y este hecho es relevante porque es justamente en la infancia (y en las instituciones y dispositivos educativos) donde se construyen y generan las miradas negativas a la diferencia. La discriminación directa o indirecta por motivos de orientación sexual está a la orden del día y es una de las principales fuentes de malestar y sufrimiento en los chicos y las chicas trans.

Se hace evidente a través de discursos y proyectos que necesitamos romper con el control biológico sobre las poblaciones, sobre los cuerpos disidentes, sobre los militantes de la diferencia. Romper con las relaciones entre pedagogía y biopolítica. Un control y un acompañamiento social y educativo que busca (mayoritariamente) producir cambios biológicos en los sujetos trans (a través de la hormonación, la cirugía, la patologización, etc. Pero la perspectiva trans situada en la dimensión transgenérica (o queer) se abre a otras dimensiones y posibilidades de ser pensada, entre las que encontramos la pedagógica. Para algunas personas trans, «lo trans» no es un problema médico, sino que se trata de un síntoma del malestar de los roles de género clásicos. Tras la hegemonía imperante del poder biomédico sobre las poblaciones, debemos buscar otras formas de afrontarlo. Y la educación es una de ellas; es una praxis con una potencia radical para transformar determinadas formas de injusticia y malestares en algunas poblaciones. Hasta hace poco las agencias sociales y educativas se mantenían cerradas en sí mismas, como formas de anquilosamiento institucional, como garantes sociales de las formas de normalidad y como actores de los medios de control de las subjetividades excesivamente perturbadoras. La necesidad y las formas como la pedagogía construyen las normatividades corporales, con respecto a la sexualidad, ha llevado a pedagogos, maestros y educadores a repensar qué hacen, cómo lo hacen y qué producen en sus praxis en relación con las temáticas sexuales.

\title{
5. Acto III: más allá de la colonización pedagógica
}

El tercer acto de nuestra investigación nos acerca a la cuestión de la colonización de las subjetividades en los procesos formativos. Nos interesa especialmente el concepto "postcolonial» porque como ocurre con muchos otros no es habitual que se le relacione con las teorías y praxis educativas y ello nos permite un sinfín de posibilidades reflexivas. Aparece frecuentemente en el campo de las humanidades, los estudios culturales, la sociología, la filosofía, etc. pero a menudo queda muy alejado del campo pedagógico. Para entender algunas de las cuestiones fundamentales de los Estudios Poscoloniales nos parece sugerente lo que propone Montserrat Galceran (2016) en un reciente trabajo:

\begin{abstract}
«La importancia de las perspectivas que voy a recorrer en este libro rebasa su condición de documentos de formas de pensar interesantes. Desestabilizan la tranquilizadora conciencia intelectual europea que, incluso con buena intención, está convencida de que la validez universal de sus doctrinas, avalada por la buena fama de que gozan en muchos centros intelectuales del mundo es a prueba de su valía. Escuchas las voces de las/los intelectuales postcoloniales y descoloniales nos advierte de que esta presencia universal del pensamiento europeo no es necesariamente la prueba de su valía intelectual, sino más bien de su poder colonial. No se impone universalmente porque sea valioso, es valioso y se impone universalmente porque es el pensamiento dominante» (p. 17).
\end{abstract}

Galceran nos sitúa, de manera clara y directa, en lo que sucede con las formas dominantes de producir e instaurar saberes. Discrepamos de ella cuando sitúa esta forma de producción de saberes sólo en Europa, porqué en el campo que nos ocupa (la educación) la producción y distribución dominante de saberes debe ubicarse también en los Estados Unidos. En educación, justamente, hemos dejado de mirar otras posiciones geográficas donde se producen saberes y que en algún momento han sido significativas: Francia e Italia, o Brasil y otros países de Latinoamérica, con producción de 
saberes pedagógicos de gran valor. Sólo por citar un contexto geográfico como el italiano, los trabajos de Costa (2018), Potestio y Togni (2011), Striano (2004), Martini (2005) o Cambi (2010) son ejemplo de ello en campos variados como la pedagogía social, la filosofía de la educación o la educación de adultos. Está claro que hemos asistido a un giro (pedagogical turn) en el enfoque de la mirada, y el contexto anglosajón domina el panorama de la producción de saberes educativos. Da a todo ello un soporte relevante el imperialismo del idioma y el control de determinadas bases de datos donde se publican la mayor parte de investigaciones vinculadas al sector educativo.

Como contraposición a este pedagogical turn, es relevante destacar lo que propone una de las principales autoras postcoloniales. Así, Gayatri Chakravorty Spivak (1999) nos dice que debemos pensar en la forma como la educación institucional o conjunto de discursos y prácticas se encuentra relacionada con la autodeterminación de las poblaciones subalternas, así como de su subordinación. Los Subaltern Studies, vinculados a la idea de postcolonialidad, han servido para sustentar nuevos discursos (la mayoría de ellos desmembrados de las tendencias dominantes) y abrir la pedagogía a otras posibilidades, a otras formas de pensar e interpretar el acto educativo mismo. Spivak (1999) termina su texto Subaltern Studies de forma contundente: «El subalterno no puede hablar. No hay virtud en las listas globales de lavandería como mujer como un artículo piadoso. La representación no se ha marchitado. La intelectual femenina como intelectual tiene una tarea circunscrita que no debe rechazar con una rúbrica» (p. 83). Los estudios postcoloniales son un eje potente para pensar, desde posiciones teóricas encarnadas, cuestiones como la formación desde la perspectiva de género, el lugar de la diferencia en las aulas, en análisis crítico de las políticas de discriminación positiva, el cuidado de sí como formas o elemento de control en la sociedad contemporánea, etc. Así es, la pedagogía puede (y debe) hacer uso de las herramientas intelectuales que le permiten repensar las formas relacionales que vehiculan los procesos de producción de alteridad. Contra el uso del lenguaje imperialista y colonial escribe Flores (2016): «Hay palabras que resultan -todavía- imposibles de escribir en pizarrones y cuadernos escolares. Palabras que manchan, que ensucian, que contagian, que no tan sólo son impronunciables sino también in-escribibles. Palabras estranguladas por las leyes de la civilización, la moral, la normalidad, la buena educación» (p. 17).

La perspectiva postcolonial sirve para intentar (con las dificultades reales que implica) que nuestra mirada sea menos europea, menos blanca, menos masculina, menos católica, menos normalizadora, más corporal y quizás, menos intelectual y también más carnal. La mirada puede transformarse, mutarse o girarse hacia otras direcciones. Es justamente a través de la mirada que ponemos en juego la metáfora que simboliza la vida misma y a través de ella se pueden romper ciertos límites. Para Han (2018) «los límites o fronteras cuya forma está determinada por la autenticidad u originalidad cultural, se disuelven. La cultura se libera, en cierto modo, de todas las costuras, limitaciones o hendiduras; pierde los límites, las barreras» (p. 21). Al romper los límites con la mirada creamos realidades y les damos sentido y forma, y es por ello que podemos pensarlas y concebirlas distintamente. La pedagogía, en clave postcolonial, trata precisamente de romper con cierta idea preconcebida sobre un ser humano a imagen y patrón de una determinada normalidad. Pero tanto para estudiantes como para profesores, no se trata de un ejercicio fácil cuando uno ha encarnado -la mayor parte de su vida- un cuerpo preestablecido. Ello requiere llevar a cabo, en los dispositivos pedagógicos, otro tipo de prácticas, tal vez "prácticas que intenten cuestionar el signo masculino/femenino a través de la transgresión. La denegación o la incorporación paródica. Ir más allá del límite manteniéndose en el límite, intentar sobrepasar el cuerpo en la insistencia de un cuerpo» (Castillo, 2015, p. 12).

Pensando en esto nos sentimos muy cómodos con la frase que escribe el filósofo Enrique Dussel (1998) en su libro Ética de la Liberación: «Ahora, pretendemos situarnos en el horizonte mundial, planetario, más allá de la región latinoamericana, de la 'heleno y euro-centrismo propio de Europa o Estados Unidos actuales, desde el centro y la periferia hacia la mundialidad» (p. 46). Intentar desplazar el centro, algunos centros, es lo que hemos procurado en este trabajo. Hay que llevar a cabo este giro, este desplazamiento hacia otras epistemologías. Y lo haremos teniendo muy presente una autora que ha marcado nuestra forma de pensar los cuerpos, Gloria Anzaldúa. La fuerza de sus palabras son ejemplo de texto-encarnado: «Me daba miedo que fuera evidente a los ojos de todo el mundo. El secreto que procuraba ocultar era que yo no era normal, que yo no era como las otras personas. Me sentía ajena, sabía que era ajena. Era la mutante a la que se expulsa del rebaño a golpes de piedra, un ser deformado con el mal de su interion» (Anzaldúa, 2016, p. 42). 


\title{
6. Conclusiones
}

Bajo esta estela de la pedagogía hemos presentado las tres categorías analíticas que en los últimos años han ocupado nuestro trabajo de investigación y docencia en la universidad y que conforman ventanas abiertas a pensar la educación desde otras dimensiones y perspectivas, con otros enfoques y posiciones. Pero el giro hacia la incorporación de toda esta perspectiva solo es posible a partir de lo que plantea Pallarés (2018):

\begin{abstract}
«La concientización ni es sólo el resultado de unas interacciones racionales ni es la consecuencia de unas prácticas educativas que han fracasado (o de otras que han triunfado) sino, más bien, es una dosificación variada que se racionaliza en análisis, consensos y compromisos que, puestos al servicio de la Pedagogía, reflejan relaciones de hechos educativos que permiten desarrollar expectativas y normas culturales compartidas entre los miembros de la comunidad escolar; hablar de hacer cambios para mejorar la educación es ya un punto de partida necesario».
\end{abstract}

Y en esa generación de cambios posibles, lo sensible explota de forma clara en muchas de las narrativas analizadas a lo largo de distintos seminarios. Se trata, de poner en juego otras dimensiones que vinculación de la subjetividad con el saber, más allá de las coordenadas cognitivas. Lo sensible pone en juego la piel, la carne, el cuerpo y le pide -a gritos- que abra sus poros para dejar traspirar sus anhelos, pero también para permitir que a través de ellos penetren micro-poros de saber. El aprendizaje no puede presentarse como algo cortado o aislado de la vida. Sobre esta cuestión insiste Foucault (2015), al hablar del papel de la filosofía en la constitución de los sujetos: «La meta de las escuelas griegas de filosofía era la transformación del individuo. La meta de la filosofía griega era dar al individuo la Capacidad que le permitiera vivir distintamente, mayor, más feliz, que otra gente» (p. 149).

La perspectiva de la transdiversidad nos abre a otras formas de entender al ser humano y los procesos formativos que lo envuelven más allá de un patrón «normativo» que pone en juego praxis normalistas. El debate entre ser normal y no serlo no es algo banal; y aunque nos pueda parecer alejado de marcos legales y políticos (como el de los derechos humanos), las lógicas rasuradoras de la diferencia operan con total impunidad. El testimonio de Andrea Achilli es muy claro y directo:

\begin{abstract}
«Llegando a la adolescencia me di cuenta de esa diferencia tan grande con las demás, sobre todo a la hora de ir al boliche, vestirse y todo eso. Odiaba los escotes, los tacos, las botas!!! Grrrrr! Terrible! Pero bueno, por un tiempo fui un poco más femenina, lo cuál duró bastante poco. Aunque ya era más grande y aún así, seguí construyéndome a mi manera y buscando mi propia satisfacción en ese mundo. En fin, niña en patas, desprolija, rotosa, despeinada, rabiosa, machona, gruñona, bruja, bocasuelta y libre» (Tron y Flores, 2013, pp. 16-17).
\end{abstract}

Pensar en la pedagogía desde la postcolonialidad implica tener presente este desplazamiento de las intenciones y del papel del sujeto educando. Se trata de dotarlo de los elementos que lo hagan, siempre en cierta medida, un sujeto autónomo, pensante y con capacidad de dirigir moralmente su vida (sin la necesidad de preceptos y recetas pensadas para los demás). Se trata de pensar en el formar, de-formar y transformar, en imaginar «la emancipación como micropoética pedagógica (y) suponer un cambio en términos de conocimiento, de ubicación y posición de los cuerpos, y de relación entre las palabras» (Flores, 2016, p. 18).

\section{Referencias}

\subsection{Referencias bibliográficas}

Anzaldúa, G. E. (2016). Borderlands/ La Frontera. La nueva mestiza. Madrid, España: Capitán Swing.

Brezinka, W. (2002). Obbietivi e limiti dell'educazione. Roma, Italia: Armando.

Cambi, F. (2010). La cura di sè como proceso formativo. Bari, Italia: Laterza.

Castillo, A. (2015). Ars Disyecta. Figuras para una corpo-politica. Santiago de Chile, Chile: Palinodia. 
Certeau, M. (2006). ¿Qué es un seminario?. En C. Rico. (Coord.) Relecturas de Michel de Certeau (pp. 43 51) México DF, México: Universidad Iberoamericana.

CLADE (2014). Diversidad sexual e identidad de género en la educación. Aportes para el debate en América Latina y el Caribe. Sâo Paulo, Brasil: CLADE.

Corazza, S. M. (2002). Para uma pedagogia do inferno na Educaçâo: Nietz̧sche, Delenz̧e e outros malditos afins. Belo Horizonte, Brasil: Autêntica.

Costa, V. (2018). Fenomenología de la educación y la formación. Salamanca, España: Ediciones Sígueme.

Dussel, E. (1998). Ética de la liberación. En la edad de la globalización y de la exclusiones. México, D. F. México: Editorial Trotta, Universidad Autónoma Metropolitana-Iztapalapa (UAM-I) y Universidad Nacional Autónoma de México (UNAM).

Duverger, M. (1996). Métodos de las ciencias sociales. Barcelona, España: Ariel.

Han, B. Ch. (2018). Hiperculturalidad. Barcelona, España: Herder.

Henry, M. (2018). Encarnación. Filosofía de la carne. Salamanca, España: Ediciones Sígueme.

Finkel, D. (2008). Dar clase con la boca cerrada. Valencia, España: Universidad de Valencia.

Flores, V. (2016). Afectos, pedagogias, infancias y heteronormatividad. Reflexiones sobre el daño. Córdoba, Argentina: Bocavulvaria Ediciones.

Flores, V. (2017). La intimidad del procedimiento. Escritura, lesbiana, sur como prácticas de sí. La Plata, Argentina: Pixel Editora.

Foucault, M. (2013). La inquietud por la verdad. México D.F., México: Siglo XXI.

Foucault, M. (2015). La ética del pensamiento. Para una crítica de lo que somos. (edición crítica de Jorge Álvarez). Madrid, España: Biblioteca Nueva.

Galceran, M. (2016). La bárbara Europa. Una mirada desde el postcolonialismo y la descolonialidad. Madrid, España: Traficantes de Sueños.

Giribuela, W. \& Nieto, F. (2010). El informe social como género discursivo. Escritura e intervención profesional. Buenos Aires, Argentina: Espacio Editorial.

Martini, B. (2005). Formare ai saperi. Per una pedagogia della conoscenza. Milán, Italia: Franco Angeli.

Miralles, P. (2014). El profesor no receta bibliografía En R. Huerta \& A. Sanz (Eds.). Educación artística y diversidad sexual (pp. 155-163). Valencia, España: Publicaciones de la Universidad de Valencia.

Missé, M. (2018). A la conquista del cuerpo equivocado. Barcelona-Madrid, España: Egales.

Pallarès, M. (2018). Recordando a Freire en época de cambios: concientización y educación. Revista electrónica de investigación educativa, 20(2), pp. 126-136.

Planella, J \& Piè, A. (Coords.) (2017). Pedagogías transgénero. Bogotá, Colombia: Editorial Magisterio.

Potestio, A. \& Togni, F. (Coords.) (2011). Bisogno di cura, Desiderio di educazione. Brescia, Italia: La Scuola. 
Rosenwald, G. \& Ochberg, R. (1993). Introduction: Life Stories, Cultural Politics and Self Understanding. En G. Rosenwald y R. Ochberg (Ed.), Storied Lives: The Cultural politics of Self-understanding (pp. 3-27). New Haven, EEUU: Yale University Press.

Shilling, Ch. (2016). The Body. A Very Short Introduction. Oxford, Reino Unido: Oxford University Press.

Spivak, G. Ch. (1999). A Critique of Postcolonial Reason: Towards a History of the Vanishing Present. Cambridge, Reino Unido: Harvard University Press.

Striano, M. (2004). Introdurione alla pedagogia sociale. Roma-Bari: Laterza.

Tolokonnikova, N. (2018). El libro Pussy Riot. De la alegría subversiva a la acción directa. Barcelona, España: Ediciones Roca.

Tron, F. \& Flores, V. (comp.) (2013). Chonguitas. Masculinidades de niñas. Neuquén, Argentina: Editora la Modonga Dark.

\subsection{Textos no publicados}

Carta 1: (2015) María, participante en el Seminario Pedagogia do Sensível. Faculdade de Educaçâo, Universidade Federal de Río Grande do Sul (Porto Alegre, Brasil).

Carta 2: (2015) Carlos, participante en el Seminario Pedagogia do Sensível. Faculdade de Educaçâo, Universidade Federal de Río Grande do Sul (Porto Alegre, Brasil).

Carta 3: (2015) Danilo, participante en el Seminario Pedagogia do Sensível. Faculdade de Educaçâo, Universidade Federal de Río Grande do Sul (Porto Alegre, Brasil)

Programa del Seminario Pedagogia do Sensível. Faculdade de Educaçâo, Universidade Federal de Río Grande do Sul (Porto Alegre, Brasil). Agosto de 2015 